\title{
Training causes activation increase in parietal and temporo-parietal regions in children with developmental dyscalculia
}

Mojtaba Soltanlou ${ }^{\mathrm{a}, \mathrm{b}, \mathrm{c}, *}$, Thomas Dresler ${ }^{\mathrm{c}, \mathrm{d}}$, Christina Artemenko ${ }^{\mathrm{a}, \mathrm{c}}$, David Rosenbaum ${ }^{\mathrm{d}}$, AnnChristine Ehlis ${ }^{\mathrm{c}, \mathrm{d}}$, Hans-Christoph Nuerk ${ }^{\mathrm{a}, \mathrm{c}}$

${ }^{a}$ Department of Ps ychology, University of Tuebingen, Tuebingen, Germany

${ }^{\mathrm{b}}$ Brain and Mind Institute and Department of Ps ychology, Western University, London, Canada

${ }^{\mathrm{c}}$ LEAD Graduate School \& Research Network, University of Tuebingen, Tuebingen, Germany

${ }^{\mathrm{d}}$ Department of Psychiatry and Psychotherapy, University Hospital of Tuebingen, Tuebingen, Germany

*Correspondence:

Mojtaba Soltanlou

Brain and Mind Institute and Department of Psychology

Western Interdisciplinary Research Building

1151 Richmond Street North

London, ON N6A 5B7, Canada

$\underline{\text { mojtaba.soltanlou@gmail.com }}$

Running title: Neuroscience of arithmetic training in DD 


\section{Highlights}

- Training-specific temporo-parietal changes for simple calculation.

- Training-specific parietal changes for complex calculation.

- Stronger training effect in younger children.

- Difficulty-related modulation of neural activity after training.

- Contradictory findings as compared to similar training in TD children. 


\begin{abstract}
While arithmetic training reduces fronto-temporo-parietal activation related to domaingeneral processes in typically developing children, we do not know whether these findings also hold for children with developmental dyscalculia (DD), who seek evidenced-based educational interventions. In a within-participant design, a group of 20 children (age range $=10.42-15$ years old) with DD underwent two weeks of arithmetic training. Brain activation was measured using functional near-infrared spectroscopy (fNIRS) before and after training to assess training-related changes. Two weeks of training led to both behavioral and brain changes. Training-specific change for trained versus untrained (control) simple multiplication solving was observed as activation increase in the bilateral temporo-parietal region including angular gyrus and middle temporal gyrus. Training-specific change for trained versus untrained (control) complex multiplication solving was observed as activation increase in the bilateral parietal region including intraparietal sulcus, superior parietal lobule, and supramarginal gyrus. We observed opposite brain activation changes in children with DD due to arithmetic training as compared to the findings of a similar study in typically developing children: Two weeks of multiplication training led to brain activation decrease in the fronto-parietal network of mental calculation in typically developing children, whereas similar training led to brain activation increase in that network in children with DD. Interestingly, these opposite brain activation changes underlie a rather similar behavioral improvement as regards response time and accuracy after two weeks of training. This finding reveals is relevant in an educational context and provides valuable insights into underlying mechanisms of mathematics learning.
\end{abstract}

Keywords: Numerical cognition, Developmental dyscalculia, Mental calculation, Arithmetic training, Functional near-infrared spectroscopy, fNIRS 


\section{Introduction}

Developmental dyscalculia (DD) is a brain-based learning disorder affecting numerical and arithmetic abilities (De Smedt, Peters, \& Ghesquière, 2019; Kaufmann, Wood, Rubinsten, \& Henik, 2011). DD emerges at the early stages of development, affecting 3-6\% of children and continues into adulthood (Kucian \& von Aster, 2015). Therefore, DD harms the career perspectives, mental health, and economic status of those diagnosed, and also puts a burden on society (Gross, Hudson, \& Price, 2009; Kaufmann et al., 2013). Surprisingly, we have little knowledge about the neural mechanisms of arithmetic processing in DD and the way these mechanisms change in the face of training. This knowledge will help us to further develop brainbased educational interventions directly derived from research in children with DD, whose brain responses differ from typically developing (TD) children. Therefore, in the current study, we aim to investigate the neurocognitive mechanisms of arithmetic learning in children with DD.

Studies in TD children reported consistent findings within the neural network of arithmetic processing with a rather high similarity across studies (Arsalidou, Pawliw-Levac, Sadeghi, \& Pascual-Leone, 2018; Peters \& De Smedt, 2018), whereas neuroimaging studies in children with DD solving arithmetic tasks provide divergent and seemingly contradictory findings. The neural network of arithmetic processing consists of a widespread fronto-parietal network: the bilateral intraparietal sulcus (related to manipulation of magnitude) and the bilateral superior parietal lobule (related to visuospatial attention) and the left angular gyrus and hippocampus (related to verbal retrieval from long-term memory and attentional demands) and the prefrontal cortex (related to executive functions and cognitive demands) (Arsalidou et al., 2018; Dehaene, Piazza, Pinel, \& Cohen, 2003; Klein et al., 2016). 
One group of studies on arithmetic processing in children with DD reported higher activation (Davis et al., 2009; Rosenberg-Lee et al., 2015; Simos et al., 2008) and hyperfunctional connectivity (Jolles et al., 2016; Michels, O'Gorman, \& Kucian, 2018; Rosenberg-Lee et al., 2015) in the fronto-parietal network as compared to TD children while solving arithmetic tasks. For instance, Davis et al. (2009) found higher activation in the bilateral precentral gyri and the right insula during simple addition in children with DD as compared to TD children, suggesting that children with DD rely on less advanced strategies to solve arithmetic problems. In a similar vein, Rosenberg-Lee et al. (2015) found higher activation in the right intraparietal sulcus, bilateral fusiform gyri, right visual cortex, and the left lingual gyrus during simple addition and subtraction in children with DD as compared to TD children (see also Simos et al., 2008). While showing higher brain activation, children with DD had worse behavioral performance in simple addition and subtraction tasks than that of TD children. Kucian and von Aster (2015) explain that children with DD overuse counting strategies and finger counting, have limited arithmetic fact retrieval, and experience difficulties with both procedural and conceptual knowledge; therefore, they overuse inefficient and compensatory strategies which leads to higher brain activation than seen in TD children. The conclusion from this group of studies is that children with DD have higher but inefficient brain activation, which is accompanied by poor behavioral performance (i.e., response time and accuracy) on mental arithmetic tasks as compared to TD children.

Another group of studies on the arithmetic abilities of children with DD observed reduced activation (Ashkenazi, Rosenberg-Lee, Tenison, \& Menon, 2012; Berteletti, Prado, \& Booth, 2014; Kucian et al., 2006; Peters, Bulthe, Daniels, Op de Beeck, \& De Smedt, 2018; Schwartz, Epinat-Duclos, Leone, Poisson, \& Prado, 2018) and structural connectivity (Rotzer et al., 2008; 
Rykhlevskaia, Uddin, Kondos, \& Menon, 2009) in the fronto-parietal network as compared to TD children during arithmetic tasks. For instance, children with DD had reduced activation in the left inferior frontal gyrus, the left middle and superior temporal gyri, the right intraparietal sulcus, and the superior parietal lobule when completing simple multiplication tasks (Berteletti et al., 2014). The authors suggest impaired arithmetic mechanisms in both numerical- and language-related regions in the brains of children with DD. Ashkenazi et al. (2012) found reduced activation related to the complexity in addition in several regions, such as the intraparietal sulcus, superior parietal lobule, angular gyrus, and supramarginal gyrus in the right hemisphere, and bilaterally in the temporal and dorsolateral prefrontal cortex for children with DD as compared to TD children (see also Molko et al., 2003). Moreover, children with DD performed worse on the addition tasks than TD children. The conclusion from this group of studies is that arithmetic problem solving does not lead to the recruitment of the relevant neurocognitive resources in children with DD.

These contradictory findings are not conclusive. It is unclear whether increased or decreased brain activation during arithmetic problem solving is an advantage or disadvantage in children with DD. More importantly, the question is how children with DD learn arithmetic and whether a behavioral improvement is accompanied by increased or decreased brain activation. By identifying these brain activation changes, the covert strategies that underly arithmetic problem solving will be disclosed to better understand the deficiencies in individuals with DD. Intervention studies can provide this insight about changes in strategies and brain responses. Surprisingly, so far only one training study investigated neural activation changes during arithmetic learning in children with DD (Iuculano et al., 2015). Iuculano et al. (2015) trained 15 children with DD for 8 weeks using one-on-one tutoring focusing on efficient counting strategies 
and arithmetic fact retrieval. Before training, they observed higher activation in the bilateral dorsolateral prefrontal and the left ventrolateral prefrontal cortex, the left intraparietal sulcus, the right fusiform gyrus, and bilateral insula during simple addition problem solving in children with DD as compared to age-matched TD peers. Thus, this training study supports the first group of literature discussed above in suggesting that there is higher brain activation in children with DD as compared to TD children. Interestingly, there were no differences in brain activation between the two groups of children after training as the over-engagement of the distributed brain activation was reduced in children with DD. This reduced activation over training manifests the existent inefficient widespread activation during simple arithmetic in DD, which is unnecessary, does not lead to appropriate performance on arithmetic tasks and therefore decreases after appropriate training.

The only existing neuroimaging study on arithmetic training in children with DD (Iuculano et al., 2015) is limited to simple calculation. Complex calculation differs from simple calculation in strategy use, procedural and conceptual knowledge (Soltanlou, Artemenko, et al., 2017). Uncovering the neural mechanisms underlying complex calculation will help us to understand arithmetic learning beyond behavioral improvements in children with DD because these children struggle more with complex calculations. While behavioral training studies mainly support different interventional approaches, they do not answer the question of why children's performance during arithmetic problem solving improves. Neuroimaging studies of arithmetic training provide more specific information about changes in the covert dysfunctions, which might be related to magnitude, cognitive, or language-related processes. These changes may not be clearly demonstrated in behavioral investigations because of the compensatory but inefficient strategies that partially cover mathematical weaknesses. This knowledge would help us to 
develop better interventions that target particular mathematical weaknesses rather than an unspecific improvement in compensatory strategies in a child's behavioral performance.

Therefore, in the present within-participant study, we investigated neural activation changes before and after two weeks of simple and complex multiplication training in children with DD. This study was built upon on our recent multiplication training in TD children (Soltanlou, Artemenko, et al., 2018), with almost the same procedure. We wanted to test whether the same training would lead to similar or different brain responses in children with DD. Moreover, by training both simple and complex arithmetic problems, we could extend the findings by Iuculano et al. (2015) that trained only simple arithmetic problems, and also test for the difficulty-related modulation of neural activity (Ashkenazi et al., 2012). The difficultyrelated modulation of neural activity is expected as distinguishable brain responses to simple and complex calculation. Ashkenazi et al. (2012) reported no distinguishable neural activation patterns during simple and complex addition in children with $\mathrm{DD}$ in a single-session measurement (see also Molko et al., 2003), which is usually observed in TD children (Soltanlou, Artemenko, et al., 2017).

Training-induced changes will be evaluated using functional near-infrared spectroscopy (fNIRS), a well-suited technique for children (Soltanlou, Sitnikova, Nuerk, \& Dresler, 2018). We predict that children with DD exhibit shorter response times and make fewer errors on both simple and complex multiplication tasks after training. Following Iuculano et al. (2015), we expect reduced fronto-parietal activation after two weeks of training on simple multiplication, particularly in the right hemisphere, which is less engaged in advanced retrieval strategies. Concerning complex multiplication, two predictions can be derived from the two groups of literature discussed above. Based on the first literature group, which argued that children with 
DD have higher neural activation as compared to TD children, we would expect reduced frontoparietal activation after training in children with $\mathrm{DD}$, which is more similar to TD children. This prediction is also in line with findings on simple addition tasks for children with DD (Iuculano et al., 2015) and complex multiplication tasks for TD children (Soltanlou, Artemenko, et al., 2018). According to the second literature group, which reported reduced neural activation in children with DD, we would expect increased fronto-parietal activation after two weeks of complex multiplication training in children with DD. Additionally, we expected differentiable brain activation between simple and complex multiplication (i.e., the difficulty-related modulation of neural activity) after training, like the complexity-related brain activation seen in TD children. Note that since the current study is a follow-up of our recent multiplication training in TD children (Soltanlou, Artemenko, et al., 2018), we will descriptively compare the current findings with the findings of that study as a control group.

\section{Materials and methods}

\section{Participants}

Twenty-five children with DD participated in the study. Five children were excluded: two did not finish the study, two because of technical problems with the fNIRS recording, and one because of only wrong answers in two conditions. Hence, 20 children ( 8 girls; age $=12.30 \pm 1.13$ years old; age range $=10.42-15$ years old) were included in the analyses. The children were from grades 4 to 8 (grade 4: 1 , grade 5: 3, grade 6: 9 , grade 7: 4, grade 8: 3). All children, except two, were right-handed. They had a normal or corrected-to-normal vision. All procedures of the study were in line with the latest revision of the Declaration of Helsinki and were approved by the ethics committee of the University Hospital of Tuebingen. 
In a screening session, math ability was assessed with the Basisdiagnostik Mathematik für die Klassen 4-8 (BASIS-MATH 4-8, Moser Opitz et al., 2010). It is a standard dyscalculia screening test in German for all children in grades 4-8, which is independent of age and grade. Note, that this is the only German test that can identify numerical deficiencies up to grade 8 (McCaskey et al., 2018). The test evaluates basic mathematical knowledge, which is achieved in primary school, including basic arithmetic operations (i.e., mental arithmetic and written procedures), understanding of the decimal place-value system, counting, number line estimation, and word problems.

The critical threshold is the total raw score of $70 \pm 3$. This test uses raw scores - rather than standardized scores based on norms - so that any child who scored above the cut-off score of 73 was considered to be in the normal range of math abilities and was not included in the current study. A score of below 67 indicates that children did not understand basic classroom math content; 15 children in the current sample scored below 67. A score between 67 and 73 belongs to the tolerance zone indicating that children are at risk of difficulties to understand basic classroom math content; 5 children in the current sample scored between 67 and 73 . The cut-off score of 73 in the current study thus includes children with math difficulties and being at risk of math difficulties. Since the recruitment was specific for children with DD, mainly parents and some teachers, who subjectively considered the child in their care to have math difficulties, approached us. Three children had a clinical diagnosis of dyscalculia, and one had attentiondeficit/hyperactivity disorder. Children and their parents gave written informed consent and received an expense allowance for their participation. 


\section{Characte ris tics of participants}

A few neuropsychological tests were utilized to find the characteristics of the sample (see Table 1). Two subtests (similarities and matrix reasoning) of the German Wechsler IQ test (Hamburg-Wechsler-Intelligenztest für Kinder-IV, Petermann, Petermann, \& Wechsler, 2007) were utilized to assess intelligence. According to the literature (Kucian \& von Aster, 2015), dyscalculia is not influenced by general intelligence, and children with an average or belowaverage level of intellect experience similar difficulties with arithmetic learning (Ehlert, Schroeders, \& Fritz-Stratmann, 2012). The number of correct answers was transformed to IQ scores $(M=100, S D=15)$.

Verbal short-term and working memory, and visuospatial short-term and working memory were assessed using the letter span test and the block tapping test (Corsi, 1973), respectively. In the verbal memory task, children were asked to recall spoken sequences of consonant letters (one letter per second). The test started with sequences of three letters, which were increased by one if the child correctly recalled at least two out of three sequences. In the visuospatial memory task, the child was asked to point to cubes in the same order as the experimenter. The procedure was the same as in the letter span test. These forward spans were considered to represent short-term memory, while backward spans (i.e., inverse recall) were considered to represent working memory in each test. Note that the score of one child was missing for the backward letter span test score (not included in Table 1).

To test the reading ability of the children, the Salzburger reading test for grades 2 to 9 (Salzburger Lese-Screening für die Schulstufen 2-9, Form A1; Wimmer \& Mayringer, 2014) was used. It contains 100 short sentences and children are asked to verify whether each sentence is grammatically correct or not. They had three minutes to evaluate as many sentences as they 
could. The number of correct answers was transformed to reading scores. Two children had a reading score of below 62, which suggests they are at risk of having a reading problem (excluded from Table 1).

Table 1: Neuropsychological data.

\begin{tabular}{lrrr}
\hline & Mean & $S D$ & Min-Max \\
\hline Verbal IQ (similarities) & 102 & 15.30 & $65-125$ \\
Non-verbal IQ (matrix reasoning) & 95.5 & 20.90 & $55-120$ \\
Verbal short-term memory (letter span - forward) & 4.5 & 0.83 & $3-6$ \\
Verbal Working memory (letter span - backward) & 3.4 & 1.00 & $3-5$ \\
Visuospatial short-term memory (Corsi - forward) & 5.2 & 0.83 & $4-7$ \\
Visuospatial working memory (Corsi - backward) & 5.0 & 0.89 & $3-7$ \\
Reading (Salzburger reading test) & 83.1 & 13.40 & $62-105$ \\
Mathematics knowledge (BASIS-MATH) & 58.7 & 10.50 & $25-73$ \\
\hline
\end{tabular}

Note: $N=20$ (but 19 for the letter span - backward, and 18 for the reading test).

\section{Procedure}

In a within-participant experiment, we measured behavioral performance and brain activation of children with DD during multiplication problem solving before and after training (Figure 1A). The experiment was conducted in a light-attenuated room and began with four practice trials. The multiplication problems were presented on the screen and the children were instructed to speak their answers as quickly and accurately as possible and then to immediately press the space-key (Figure 1A). The experimenter, who was sitting behind the child, wrote down the answers on a sheet. Each problem was randomly presented once during the pre-test and the post-test. Each problem was presented until keypress or for a maximum duration of $60 \mathrm{~s}$, and was followed by 20 s of rest (i.e., inter-trial interval; Figure 1A). Due to individual differences in reaction times, the presentation of problems differed both within and between children in the current self-paced event-related design. No feedback was given during the experiment. 
After the pre-test, six similar training sessions were performed through an online learning platform (Soltanlou, Jung, et al., 2017) at home for two weeks. In the post-test, children were measured again to evaluate training effects. The problems were identical for each condition in pre- and post-test sessions. The experiment was run using the software Presentation (version 16.3, NeuroBehavioral Systems, Inc., Berkeley, USA). This study was part of a combined fNIRS-EEG project, and only the fNIRS findings are reported here.

A

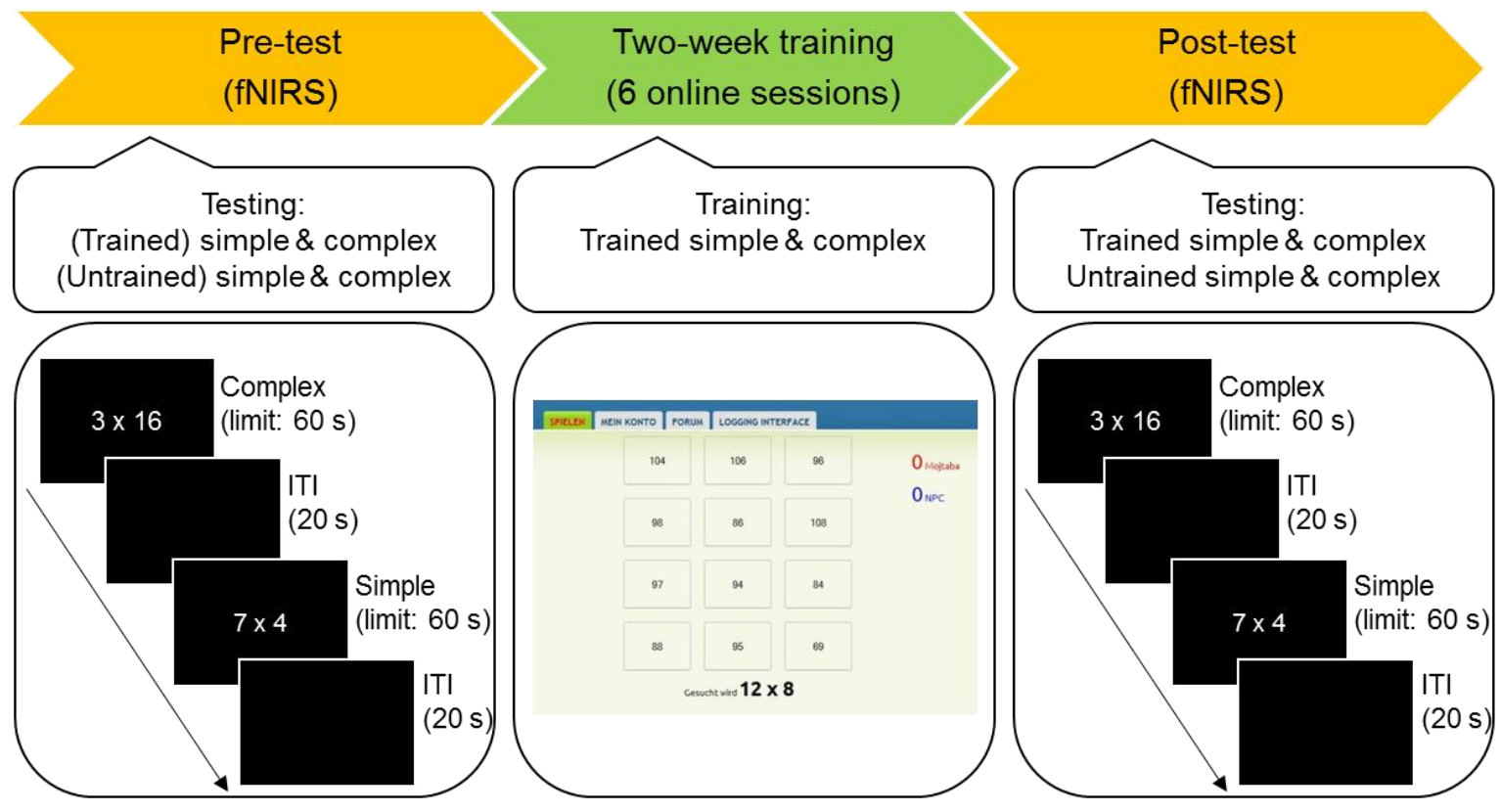

B

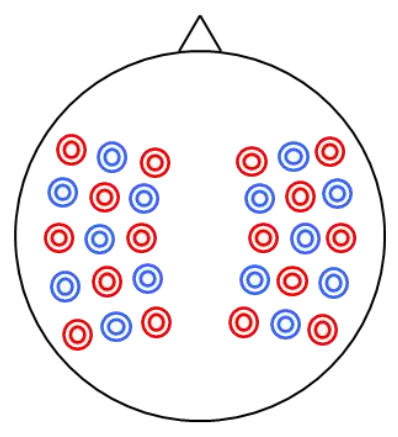

C

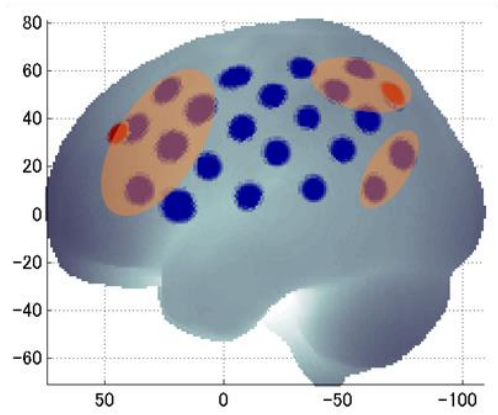

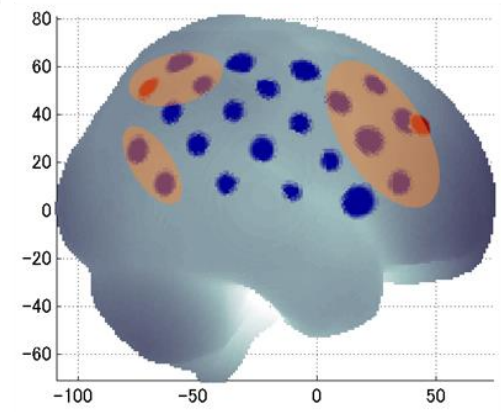

Figure 1: A) Procedure of the study showing the pre-post design with fNIRS recordings and the online training of multiplication. B) Schematic positions of fNIRS optodes. Red circles indicate emitters and blue circles indicate detectors in the two $3 \times 5$ arrays. $C$ ) fNIRS channels layout (by 
Minako Uga). Blue circles indicate the fNIRS channels projected on the brain surface. Red circles indicate $\mathrm{P} 3 / \mathrm{P} 4$, and $\mathrm{F} 3 / \mathrm{F} 4$ positions - according to the international 10-20 system projected on the brain surface. Channels included in the parietal, frontal, and temporo-parietal ROIs are marked by the orange ovals (see also Artemenko et al., 2018).

\section{Neuroimaging task}

In the present study, 16 simple and 16 complex multiplication problems were used, the same as in our training study in TD children (Soltanlou, Artemenko, et al., 2018). Half of the problems in each set were allotted for training, and the other, closely matched half were used as untrained problems in the pre- and post-test only, resulting in four conditions: trained simple, untrained simple, trained complex, and untrained complex (for the list of the items see Supplementary Material, Table S7). Simple problems (e.g., $3 \times 7$ ) included two single-digit operands (range 2-9) with a two-digit solution (range 12-40). Complex problems (e.g., 4×19) included a two-digit (range 12-19) multiplied by a single-digit operand (range 3-8) with a twodigit solution (range 52-98). In half of the items in each condition, the first operand was larger than the second operand.

\section{Inte ractive online training}

The training was done using an online learning platform (Soltanlou, Artemenko, et al., 2018; Soltanlou, Jung, et al., 2017), which allowed for at-home training (Kesler, Sheau, Koovakkattu, \& Reiss, 2011; Kucian et al., 2011). The children participated in six training sessions over two weeks in their homes (Figure 1A). Children were trained only for the trained simple and trained complex multiplication sets and not for the untrained sets. The multiplication problems within each condition were presented randomly in each run, for a total of five runs in each training session (i.e., a total of 80 problems per session). Each problem was individually 
presented along with 12 different choices including the correct solution. Detailed description of the choices can be found elsewhere (Soltanlou, Artemenko, et al., 2018). Response intervals for simple problems ranged randomly from $7-20 \mathrm{~s}$, jittered by $1.3 \mathrm{~s}$, and for complex problems from 15-45 s, jittered by $3 \mathrm{~s}$. In an interactive competition, the computer displayed the correct solution whenever the child did not respond within the response interval. To provide feedback on performance and to increase motivation, the scores of the child and the computer were shown on the right side of the screen (Soltanlou, Artemenko, et al., 2018). Both the child and the computer received one point for each correct answer and one point was deducted for each incorrect answer. The problem was presented until the child responded correctly or correct answer was given. To create a more realistic competition and to increase participant motivation, the computer responded incorrectly for $30 \%$ of the problems. Children were instructed to solve the problems as quickly and accurately as possible.

\section{fNIRS recording and preprocessing}

fNIRS data were collected with the ETG 4000 Optical Topography System (Hitachi Medical Corporation, Tokyo, Japan) using two wavelengths of $695 \pm 20 \mathrm{~nm}$ and $830 \pm 20 \mathrm{~nm}$ to measure the absorption changes of oxygenated $\left(\mathrm{O}_{2} \mathrm{Hb}\right)$ and deoxygenated $(\mathrm{HHb})$ hemoglobin, according to the modified Beer-Lambert law. The data were recorded with a $10 \mathrm{~Hz}$ sampling rate, the fixed inter-optode distance was $30 \mathrm{~mm}$. Using two $3 \times 5$ arrangements of optodes ( 8 emitters and 7 detectors each, Figure 1B) in an elastic combined fNIRS-EEG cap (Brain Products $\mathrm{GmbH}$, Herrsching, Germany) resulted in 22 measurement channels for each hemisphere (Figure 1C). The correspondence of the fNIRS channels to the underlying cortical regions was estimated based on a virtual registration method (Rorden \& Brett, 2000; Singh, 
Okamoto, Dan, Jurcak, \& Dan, 2005; Tsuzuki et al., 2007) and labeled according to the automated anatomical labeling (AAL) atlas (Tzourio-Mazoyer et al., 2002). For more details see Soltanlou, Artemenko, et al. (2017).

Continuous changes in the concentration of $\mathrm{O}_{2} \mathrm{Hb}$ and $\mathrm{HHb}$ were recorded for all channels during the measurements. These changes occur through neurovascular coupling in response to cortical activation. Data were preprocessed and analyzed with custom MATLAB routines (The MathWorks, Inc., USA). The continuous signals were bandpass filtered with $0.01-0.2 \mathrm{~Hz}$ to remove long-term drift of baseline, and high-frequency cardiac and respiratory activities (Haeussinger et al., 2014; Sasai, Homae, Watanabe, \& Taga, 2011; Scholkmann et al., 2014; Tong \& Frederick, 2010). The remaining noisy channels (7.5\%) were detected by visual inspection for each participant and interpolated using the average signal of surrounding channels. Most of these channels were located on bilateral temples and were not included in the regions of interest (ROIs; see below). Incorrect and missing trials (10.5\%) were excluded. To address possible motion artifacts, which is particularly important with children, and to reduce nonevoked systemic influences (Haeussinger et al., 2014; Scholkmann et al., 2014), we used the correlation-based signal improvement (CBSI) method (Cui, Bray, \& Reiss, 2010), as one of the recommended artifact correction methods in fNIRS signal processing (Brigadoi et al., 2014; Fishburn, Ludlum, Vaidya, \& Medvedev, 2019). The CBSI time course, which is calculated based on the assumed negative correlation between concentrations of $\mathrm{O}_{2} \mathrm{Hb}$ and $\mathrm{HHb}$, was used for further analysis. Using a data-driven approach based on the grand average of the fNIRS time series of all correctly solved trials across all channels, participants, and conditions, the amplitude of $10 \mathrm{~s}$ after stimulus onset (i.e., 0-10 s) was averaged for each channel, participant, and condition. The grand average revealed that the rising signal came back to the baseline at $10 \mathrm{~s}$ 
after the stimulus onset. This average was baseline-corrected using the $5 \mathrm{~s}$ before each respective trial and used for the analysis.

\section{Analysis}

\section{Behavioral}

Response times (RTs) were defined as the time interval from problem presentation until participant keypress after the verbal answer in the pre- and post-measurements. Median RTs were calculated based on correct responses only (89.5\% of problems across pre- and post-test). The error rate was defined as the proportion of incorrect and missing responses to the total number of presented trials. Separate repeated-measures analyses of covariance (rmANCOVAs) were conducted for median RTs and error rates, and for simple and complex multiplication. Each $2 \times 2$ rmANCOVA comprised the within-factors of measurement time (pre- versus post-test), and training (trained versus untrained) and grade as a continuous covariate because of its rather wide range. According to the literature, having a greater amount of education makes it easier to solve arithmetic problems, and different educational levels lead to differences in performance and brain activation patterns (Artemenko, Soltanlou, Ehlis, Nuerk, \& Dresler, 2018; McCaskey et al., 2018; Ranpura et al., 2013). We further conducted correlation analyses to test whether children's performance in the pre-test predicts the training effect (i.e., changes in RT and error rate over time by calculating the difference between these variables in pre- and post-test).

fNIRS

In the first step, ROIs were defined within the neural network for arithmetic processing. They consisted of the frontal (bilateral middle frontal gyrus and inferior frontal gyrus), parietal 
(bilateral intraparietal sulcus, superior parietal lobule, and supramarginal gyrus), and temporoparietal (bilateral angular gyrus and middle temporal gyrus) cortices (Figure $1 \mathrm{C}$ ). The $2 \times 2 \times 2$ rmANCOVA comprised the within-factors of measurement time (pre- versus post-test), training (trained versus untrained), hemisphere (left versus right), and grade as a covariate. The rmANCOVAs were conducted for each ROI, separately for simple and complex multiplication.

In the next step, to test for the difficulty-related modulation of neural activity due to multiplication training, a global analysis was conducted. All 44 channels covering both hemispheres were defined as one ROI. The $2 \times 2$ rmANCOVA comprised the within-factors of measurement time (pre- versus post-test), complexity (simple versus complex), and grade as a covariate. The statistical analyses were completed using R (R Core Team, 2018) and jamovi (Version 1.1.9).

\section{Results}

\section{Behavioral}

Simple

The rmANCOVA of median RT for simple multiplication revealed no significant effects (Figure 2A). The rmANCOVA of error rate revealed a significant main effect of training $\left[F(1,18)=6.52, p=.020, \eta_{p}{ }^{2}=.266\right]$, showing that the children made fewer errors when solving trained simple problems than untrained simple problems (Figure 2B). Further analysis revealed that the main effect of training comes from the post-test difference between trained and untrained simple problems $[t(33.24)=2.22, p=.033]$, while there was no significant difference in the pretest $[t(33.24)=1.37, p=.179]$. This observation suggests training-specific improvement as regards error rate in simple conditions. A significant interaction of training $\times$ grade $[F(1,18)=$ 
4.90, $\left.p=.040, \eta_{p}{ }^{2}=.214\right]$ indicated a larger difference between trained and untrained conditions for older children $[r(18)=.46, p=.040]$. Other effects were not significant (see Supplementary Materials, Table S2).

A

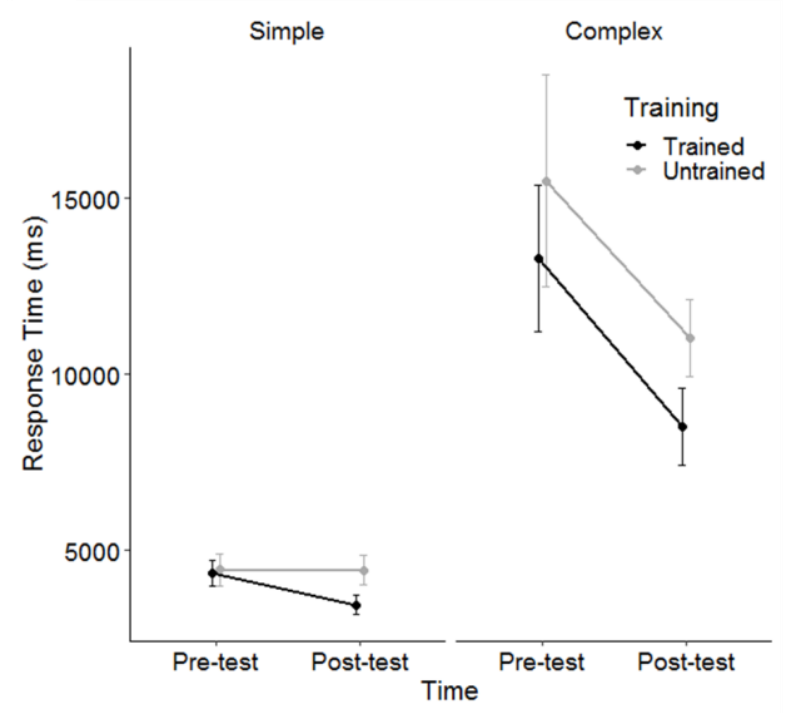

B

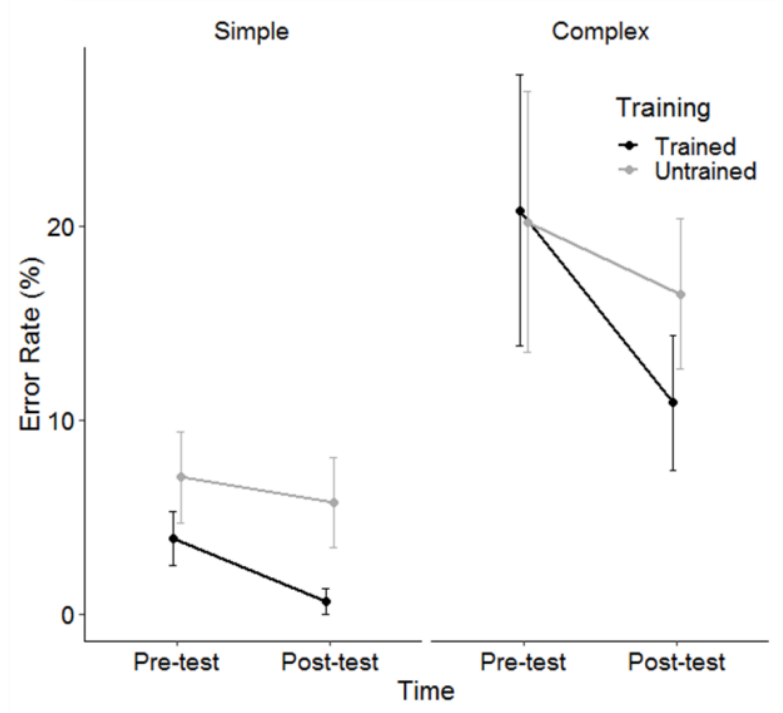

Figure 2: A) Median RT and B) Error rate for simple and complex multiplication pre-test and post-test after training on a portion of problems. Error bars depict 1 standard error $(S E)$ of the mean. For simplicity, the values in the figure are not adjusted for the covariate.

\section{Complex}

The rmANCOVA of median RT for complex multiplication revealed a significant main effect of time $\left[F(1,18)=5.39, p=.032, \eta_{p}{ }^{2}=.230\right]$, showing that the children became faster at complex multiplication after training (Figure 2A). This observation suggests training improved performance in both trained $[t(29.59)=2.61, p=.014]$ and untrained $[t(29.59)=2.43, p=.022]$ complex problems over time. The significant main effect of training $[F(1,18)=4.59, p=.046$, $\left.\eta_{p}{ }^{2}=.203\right]$ indicated faster responses for trained complex problems than untrained complex problems. Further analysis revealed that the main effect of training comes from the post-test 
difference between trained and untrained complex problems $[t(33.73)=2.12, p=.041]$, while there was no significant in the pre-test $[t(33.24)=1.84, p=.074]$. This observation suggests training-specific improvement as regards median RT in complex conditions. Furthermore, the significant main effect of grade $\left[F(1,18)=14.70, p=.001, \eta_{p}{ }^{2}=.450\right]$ indicated that older children responded faster than younger children $[r(18)=-.67, p=.001]$.

The rmANCOVA of error rate revealed a significant main effect of time $[F(1,18)=4.39, p$ $\left.=.050, \eta_{p}{ }^{2}=.196\right]$, showing that the children made fewer errors after training (Figure 2B). Further analysis revealed a marginal effect of time in trained complex problems $[t(27.47)=1.83$, $p=.078]$, but not in untrained complex problems $[t(27.47)=0.69, p=.499]$. The main effect of grade $\left[F(1,18)=11.60, p=.003, \eta_{p}^{2}=.391\right]$ indicated that older children made fewer errors than younger children $[r(18)=-.63, p=.003]$. Other effects were not significant (see Supplementary Materials, Table S2).

The correlation analysis between the RTs and error rates before training predicted the training effect (trained simple RT: $r(18)=.70, p<.001$; trained complex RT: $r(18)=.91, p<$ .001 ; trained simple error rate: $r(18)=.92, p<.001$; trained complex error rate: $r(18)=.88, p<$ .001). This finding suggests that children with poorer performance before training gained more from the two weeks of multiplication training.

\section{fNIRS}

\section{Simple}

The rmANCOVA revealed only a significant interaction of time $\times$ training in the temporoparietal cortex $\left[F(1,18)=4.72, p=.043, \eta_{p}{ }^{2}=.208\right]$, suggesting training-specific changes. Whereas temporo-parietal activation increased during trained simple multiplication solving after 
two weeks, this activation decreased during untrained simple multiplication solving (Figure 3A). Other effects were not significant (see Supplementary Materials, Table S3).

A

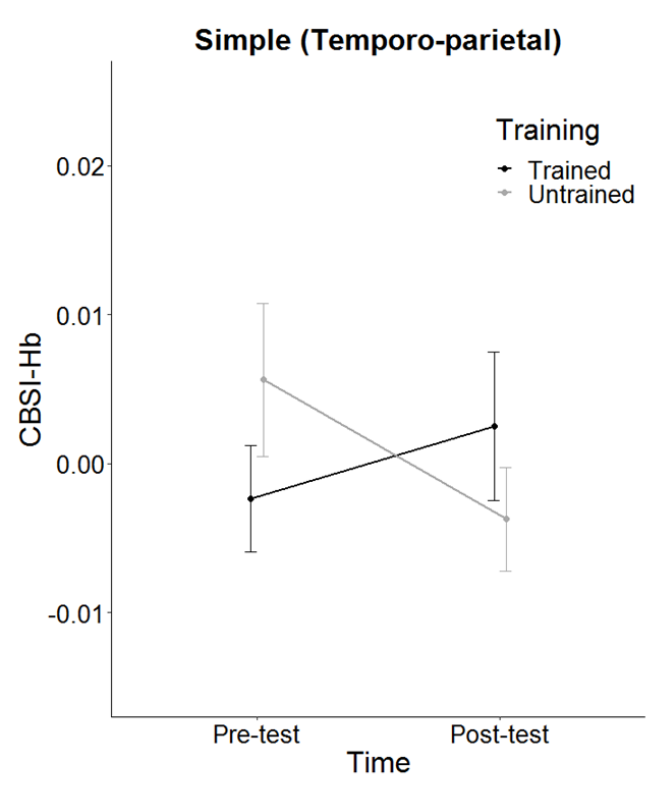

C

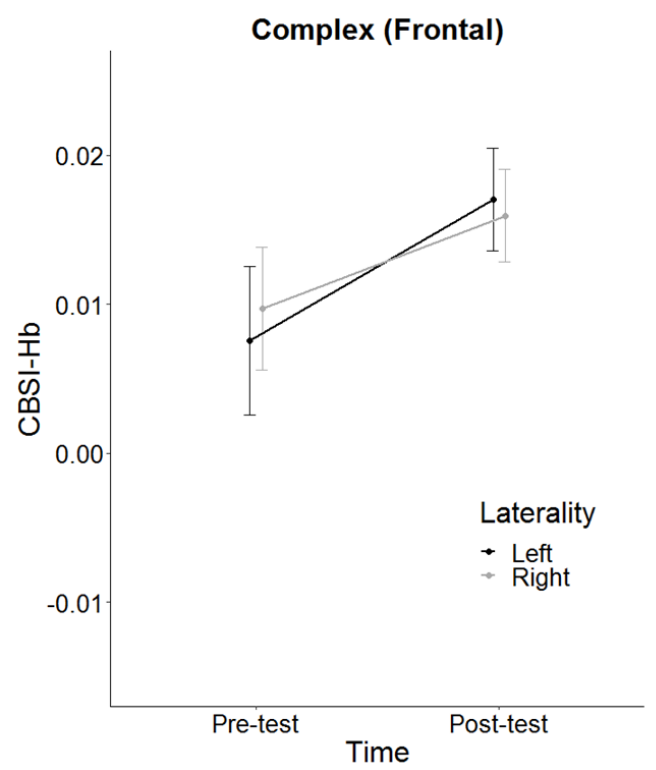

B

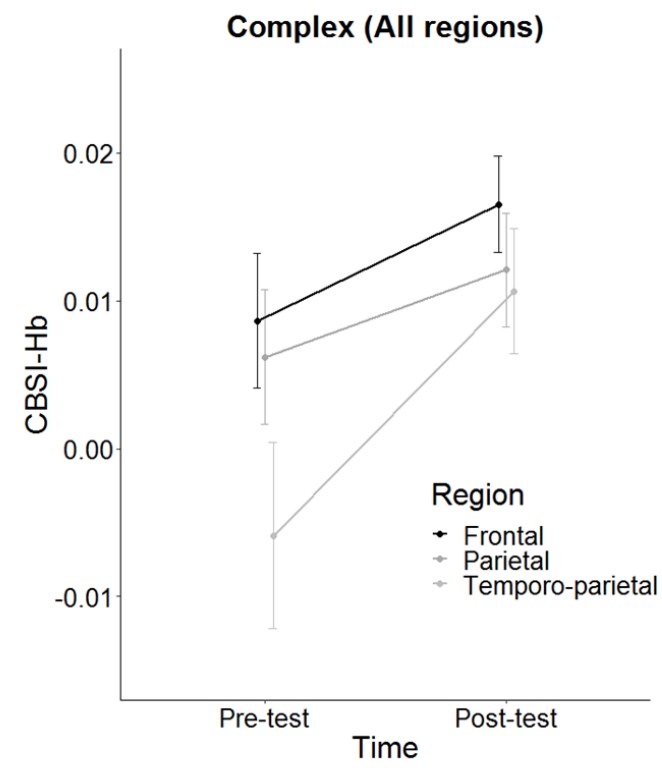

D

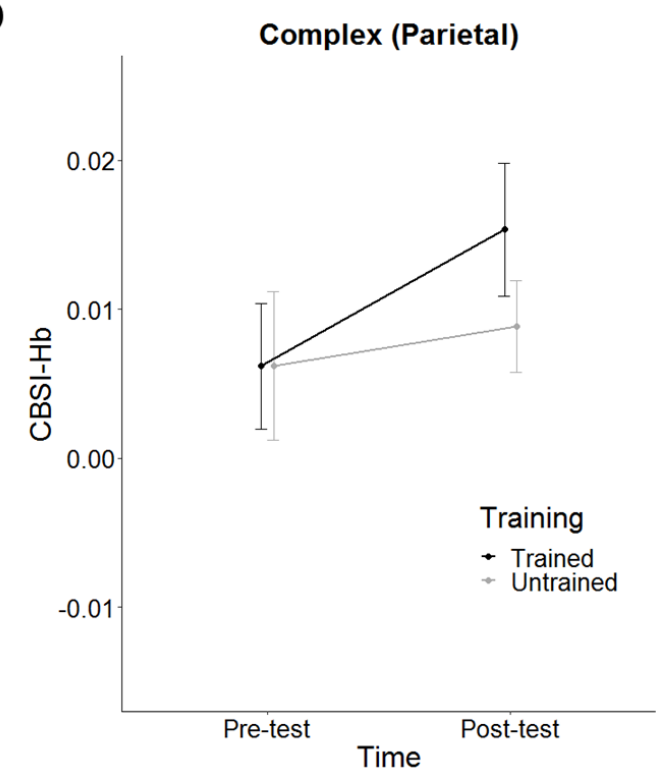

Figure 3: A) Temporo-parietal activation increased for trained simple multiplication problems after two weeks of training and decreased for untrained simple multiplication problems. B) Activation in frontal and temporo-parietal cortices, but not in the parietal cortex, significantly increased for complex multiplication problems after two weeks of training. C) The activation increase in the left frontal cortex was significantly greater than in the right frontal cortex after two weeks of training. D) Parietal activation significantly increased for trained complex multiplication problems after two weeks of training as compared to untrained complex 
multiplication problems. Error bars depict $1 S E$ of the mean. For simplicity, the values in the figure are not adjusted for the covariate.

\section{Complex}

In the frontal cortex, a significant main effect of time $\left[F(1,18)=6.46, p=.020, \eta_{p}{ }^{2}=.264\right]$ and an interaction of time $\times$ grade $\left[F(1,18)=5.42, p=.032, \eta_{p}^{2}=.231\right]$ (Figure 2B) showed increased activation after two weeks of training, particularly in younger children $[r(18)=-.48, p$ $=.032]$. The significant interaction of time $\times$ hemisphere $\left[F(1,18)=6.37, p=.021, \eta_{p}^{2}=.261\right]$ and the interaction of time $\times$ hemisphere $\times$ grade $\left[F(1,18)=5.77, p=.027, \eta_{p}{ }^{2}=.243\right]$ showed a greater increase in the left frontal activation than in the right frontal activation after two weeks of training (Figure 3C), particularly in younger children.

In the parietal cortex, a significant main effect of hemisphere $\left[F(1,18)=5.14, p=.036, \eta_{p}^{2}\right.$ $=.222]$ and an interaction of hemisphere $\times \operatorname{grade}\left[F(1,18)=5.20, p=.035, \eta_{p}^{2}=.224\right]$ showed greater activation in the right than in the left parietal cortex, particularly in younger children $[r(18)=.47, p=.035]$. The significant interaction of time $\times$ training $[F(1,18)=5.63, p=.029$, $\left.\eta_{p}{ }^{2}=.238\right]$ and the interaction of time $\times$ training $\times \operatorname{grade}\left[F(1,18)=5.12, p=.036, \eta_{p}{ }^{2}=.221\right]$ revealed training-specific changes. The parietal activation increased during trained complex multiplication after two weeks of training as compared to untrained complex multiplication (Figure 3D), particularly in younger children.

In the temporo-parietal cortex, a significant main effect of time $[F(1,18)=6.21, p=.023$, $\left.\eta_{p}{ }^{2}=.256\right]$ showed increased activation after two weeks of training (Figure 3B). Other effects were not significant (see Supplementary Materials, Table S4). 


\section{Global analysis}

To test difficulty-re lated modulation of neural activity, the rmANCOVA on global activation revealed a significant interaction of time $\times$ complexity $\left[F(1,18)=4.84, p=.041, \eta_{p}^{2}=\right.$ .212], showing increased activation only for complex multiplication after two weeks of training $[t(35.96)=2.06, p=.047]$ (Figure 4). Other effects were not significant (see Supplementary Materials, Table S5).

A
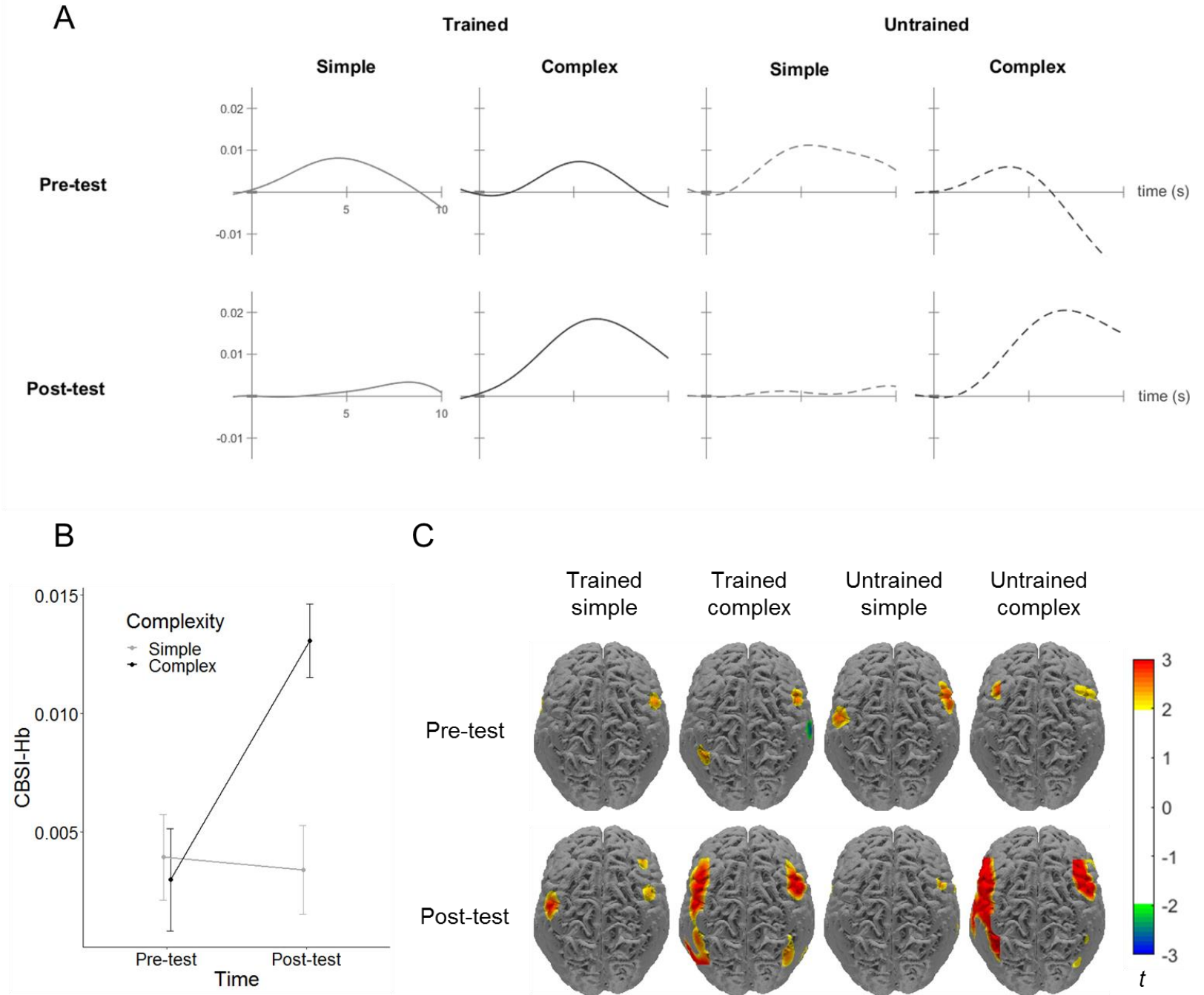

C

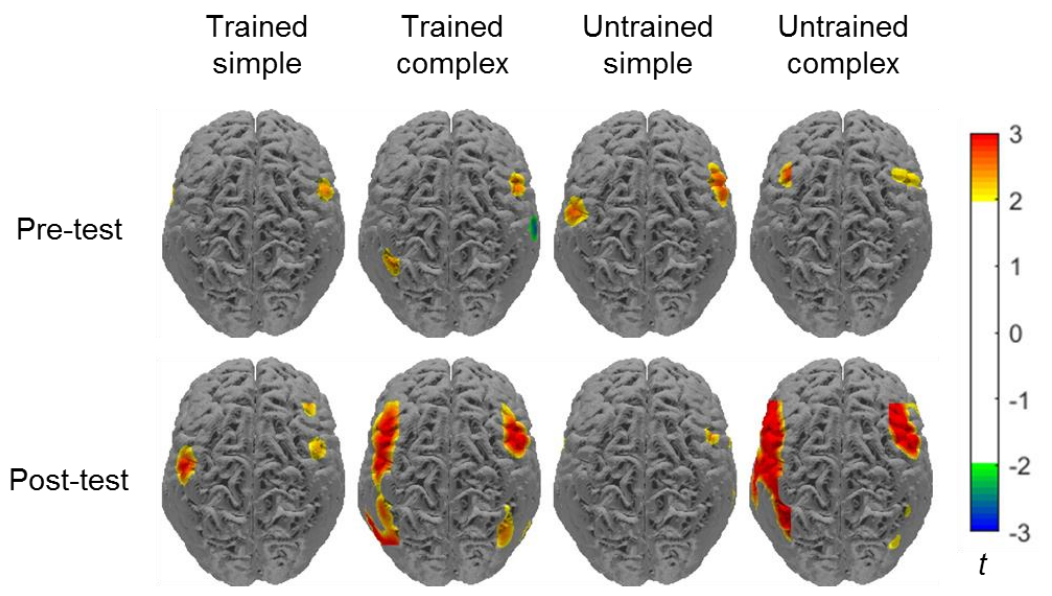

Figure 4: Difficulty-related modulation of neural activity: A) The time course of global activation (i.e., mean activation of all 44 channels) for each condition in pre- and post-test. Simple and complex conditions are distinguishable in the post-test but not in the pre-test. B) 
Global activation for simple (both trained and untrained) and complex (both trained and untrained) conditions in pre- and post-test. Error bars depict $1 S E$ of the mean. For simplicity, the values in the figure are not adjusted for the covariate. C) The heat map of brain activation for each condition in pre- and post-test. Red and blue demonstrate increased and decreased activation, respectively.

\section{Discussion}

The present study aimed at uncovering the brain activation changes underlying arithmetic learning in children with DD. Children with DD underwent computer-based arithmetic training for two weeks. In a within-participant design, brain activation in children with DD was measured using fNIRS during simple and complex multiplication problem solving before and after the training, following up on our previous study in TD children (Soltanlou, Artemenko, et al., 2018).

\section{Ne urobehavioral changes during simple calculation in children with DD}

On the behavioral level, fewer errors were observed in the trained simple as compared to untrained simple problems in the post-test, showing that two weeks of training was effective (see also Supplementary Materials, Table S6 for transfer effects). On the neural level, two weeks of training led to training-specific changes: temporo-parietal activation increased for trained simple multiplication problems but decreased for untrained simple multiplication problems. The left angular gyrus of the temporo-parietal cortex is a part of the arithmetic fact retrieval network and automatization (Dehaene et al., 2003; Klein et al., 2016). Our training probably led to the engagement of this network during simple multiplication problem solving, which indicates that the children used advanced strategies, such as rote retrieval strategy and automatized procedures, to a greater degree. The increased temporo-parietal activation for trained simple multiplication supports previous studies showing reduced overall brain activation in children with DD as compared to TD children in a single-session measurement (Berteletti et al., 2014). 
While the left angular gyrus has frequently been associated with multiplication fact retrieval, the literature on the role of the right angular gyrus is less conclusive. Arsalidou and Taylor (2011) suggested that the right angular gyrus is involved in goal-directed salience representations and supports visuospatial attentional demands during mental arithmetic. Altogether, we might conclude that children with DD became more fluent and automatized in their problem solving strategies only for the simple multiplication problems on which they received direct training but they could not transfer this fluency to the solving of untrained simple multiplication problems.

\section{Ne urobehavioral changes during complex calculation in children with DD}

Children with DD improved in their behavioral performance in trained as compared to untrained complex arithmetic after training (see also Supplementary Materials, Table S6 for transfer effects). On the neural level, training-related activation changes were exclusively observed in the parietal region and generalized to untrained complex multiplication in the frontal and temporo-parietal regions (Figure 3B and 3D). It seems that children with DD learned how to apply procedural steps to solve both trained and untrained complex problems after two weeks; therefore, their ability to solve both trained and untrained complex problems improved, but to a greater degree in trained problems.

As one of the most interesting findings in the current study, two weeks of arithmetic training improved the numerical and spatial processes related to mental calculation. This was reflected by the training-related increase in parietal activation, particularly in younger children. The parietal cortex, i.e., the intraparietal sulcus and the superior parietal lobule, is involved in magnitude and visuospatial attentional processes during mental arithmetic (Dehaene et al., 2003). 
The increased engagement of these regions during trained complex problem solving but not untrained complex problems supports previous literature on the dysfunction of these regions in DD. For instance, Kucian et al. (2014) suggested an impaired or delayed development of axonal coherence and myelination in the fibers projecting to/from the parietal cortex in children with DD, which might point to a disconnection syndrome (Molko et al., 2003; Molko et al., 2004). We may conclude that two weeks of training partially resolved this disconnection syndrome and reduced the arithmetic deficit in children with DD.

We also observed higher activation in the right than in the left parietal cortex, particularly in younger children. While the right intraparietal sulcus is engaged in non-symbolic number magnitude processing, particularly at early ages (Edwards, Wagner, Simon, \& Hyde, 2016; Hyde, Boas, Blair, \& Carey, 2010), the left intraparietal sulcus is deeply involved in symbolic number magnitude processing of the numbers at school ages (Vogel, Goffin, \& Ansari, 2015) in association with language-related areas on the left hemisphere. Supporting our current finding, the right parietal cortex is expected to be involved in the calculation to a greater degree in younger children than the left parietal cortex, according to a recent theoretical model of functional lateralization in the parietal cortex in arithmetic (Artemenko, Sitnikova, Soltanlou, Dresler, \& Nuerk, 2020).

Two weeks of training improved the cognitive processes related to mental calculation, as frontal activation generally increased after training, particularly in the left hemisphere. This improvement was not training-specific and observed in both trained and untrained complex problem solving. Frontal activation within the fronto-parietal network is associated with domaingeneral cognitive processes during complex arithmetic (Klein et al., 2016). These frontal circuits are mainly involved in procedural strategies, which are the strategies usually applied by TD 
children for complex arithmetic (Arsalidou et al., 2018; Peters \& De Smedt, 2018). This frontal activation increase was higher for younger children, who exhibited poorer performance than older children before training. Therefore, it is likely that younger children gained more from training (see also McCaskey et al., 2018).

Two weeks of training improved the language-related and goal-directed processes related to mental calculation, as reflected by an activation increase in the temporo-parietal region, i.e., angular gyrus and middle temporal gyrus. Similar to the frontal activation increase, this improvement was not training-specific and observed for both trained and untrained complex problem solving. The left and right angular gyrui are respectively engaged in language-related processes (Kesler et al., 2011) and goal-directed salience representations during mental arithmetic (Arsalidou \& Taylor, 2011). The increased involvement of these processes seems rational because, among the basic arithmetic operations, multiplication is the one that strongly relies on verbal repetitions and even complex multiplication usually is decomposed into simple facts that might be retrieved from memory. It seems that children with DD develop retrieval and automated procedural strategies, which lead to increased bilateral angular gyrus activation (Ashkenazi, Black, Abrams, Hoeft, \& Menon, 2013; Polspoel, Peters, Vandermosten, \& De Smedt, 2017; Rykhlevskaia et al., 2009). It is worth noting that one of the most common procedures in complex calculation is to split the calculation into small steps which can be solved by retrieval or fast procedural processes (Soltanlou, Artemenko, et al., 2018). Therefore, retrieval processes are part of procedural strategies used during complex multiplication and increased bilateral angular gyrus activation within the temporo-parietal region is interpreted as a sign of training effectiveness. This interpretation is supported by higher retrieval strategies after two weeks of training as compared to before training (see Supplementary Material, Table S6). 


\section{Difficulty-related modulation of neural activity}

Two weeks of training led to the difficulty-related modulation of neural activity in children with DD (Ashkenazi et al., 2012; Molko et al., 2003): discriminable brain activation for simple versus complex multiplication problem solving was observed after training but not before training. This reflects the more extended brain activation pattern during complex than simple arithmetic, which is typically observed for TD children (e.g., Soltanlou, Artemenko, et al., 2017). Note that although this difficulty-related modulation effect is widespread, it is not simply a systemic effect because it comes from an interaction effect, where increased activation is only observed for complex multiplication and not simple multiplication, and the simple and complex problems were randomly presented during the current event-related design.

The development towards typical arithmetic processing by children with DD is supported by other training studies (Iuculano et al., 2015; Michels et al., 2018). Iuculano et al. (2015) found that brain activation in children with DD is significantly discriminable from TD peers only before, not after, training. In the same vein, Michels et al. (2018) reported discriminable functional connectivity between children with DD and TD children before, but not after, five weeks of number line training. The data from the current study indicate that two weeks of multiplication training activate the most relevant neural networks for arithmetic processing so that brain activation patterns for children with DD are like those of TD children from other studies.

\section{Limitations and future perspectives}


We acknowledge our rather small sample size and broad age range in the current study and we are aware of the replicability crisis in psychology and neuroscience. However, we should mention that data collection in clinical populations is quite challenging and time-consuming. To overcome this barrier, and some others such as different DD definition criteria, comorbidity and heterogeneity, experimental design and data-analytic methods (De Smedt et al., 2019; Kaufmann et al., 2013), we recommend multi-lab and multivariate approaches with a large $N$ for future research. Moreover, in the absence of a direct control group within the same statistical models, the current study followed our previous multiplication training study in typically developing children (Soltanlou, Artemenko, et al., 2018) and results were compared between these studies. As a methodological limitation, fNIRS with its restricted depth resolution only allows for the measurement of about $1-1.5 \mathrm{~cm}$ of the cortex (corresponding to $3 \mathrm{~cm}$ from the scalp) in adults (Haeussinger et al., 2011; Schroeter, Kupka, Mildner, Uludağ, \& von Cramon, 2006). Therefore, we were not able to record activation changes in subcortical regions such as the hippocampus that are involved in mental arithmetic (e.g., Klein et al., 2016).

\section{Conclusion}

This study followed our recent multiplication training in TD children (Soltanlou, Artemenko, et al., 2018) and investigated arithmetic learning in children with DD. A two-week training by an interactive learning platform improved behavioral arithmetic performance in both TD children and children with DD. Surprisingly, opposite brain activation changes were observed in TD children (Soltanlou, Artemenko, et al., 2018) and in children with DD (current study): While two weeks of multiplication training led to brain activation decrease in the frontoparietal network of mental calculation in TD children, the same training led to brain activation increase in that network in children with DD. We conclude that (i) the neural mechanisms of 
arithmetic learning differ between TD children and children with DD, (ii) while calculation improvement relates to more automatized and less effortful processes in TD children, this improvement relates to more and functionally better usage of cognitive processes in children with DD, (iii) the application of neuroimaging in the educational context provides valuable insights about the underlying mechanisms of mathematics learning (Dresler et al., 2018), (iv) educational interventions should develop based on the direct findings in individuals with DD rather than generalizations of findings in typical populations. 


\section{Acknowledgment}

We would like to thank all participating children and their parents, and our assistants who helped with data collection and proofreading the manuscript. This research was funded by a grant from the Science Campus Tuebingen, project 8.4 to HCN supporting MS. MS is the recipient of a BrainsCAN Postdoctoral Fellowship at Western University, funded by the Canada First Research Excellence Fund (CFREF). MS, TD, CA, ACE, and $\mathrm{HCN}$ are members of the LEAD Graduate School \& Research Network [GSC1028], which is funded within the framework of the Excellence Initiative of the German federal and state governments.

\section{Author contributions}

MS, TD, and HCN designed and conceptualized the study. MS collected and analyzed data, and drafted the manuscript. All authors reviewed and revised the manuscript.

\section{Data Availability Statement}

The data that support the findings of this study are available on request from the corresponding author. The data are not publicly available due to privacy or ethical restrictions.

\section{Supplementary Material}

This article contains supplementary material.

\section{Conflict of interest}

The authors declare no conflict of interest. 


\section{References}

Arsalidou, M., \& Taylor, M. J. (2011). Is 2+2=4? Meta-analyses of brain areas needed for numbers and calculations. Neuroimage, 54(3), 2382-2393.

Arsalidou, M., Pawliw-Levac, M., Sadeghi, M., \& Pascual-Leone, J. (2018). Brain areas associated with numbers and calculations in children: Meta-analyses of $\mathrm{fMRI}$ studies. Developmental Cognitive Neuroscience, 30, 239-250.

Artemenko, C., Soltanlou, M., Ehlis, A. -C., Nuerk, H.-C., \& Dresler, T. (2018). The neural correlates of mental arithmetic in adolescents: a longitudinal fNIRS study. Behavioral and brain functions, 14(1), 5.

Artemenko, C., Sitnikova, M. A., Soltanlou, M., Dresler, T., \& Nuerk, H. -C. (2020). Functional lateralization of arithmetic processing in the intraparietal sulcus is associated with handedness. Scientific reports, 10(1), 1775.

Ashkenazi, S., Rosenberg-Lee, M., Tenison, C., \& Menon, V. (2012). Weak task-related modulation and stimulus representations during arithmetic problem solving in children with developmental dyscalculia. Developmental Cognitive Neuroscience, 2 Suppl 1, S152-166.

Ashkenazi, S., Black, J. M., Abrams, D. A., Hoeft, F., \& Menon, V. (2013). Neurobiological underpinnings of math and reading learning disabilities. Journal of Learning Disabilities, 46(6), 549-569.

Berteletti, I., Prado, J., \& Booth, J. R. (2014). Children with mathematical learning disability fail in recruiting verbal and numerical brain regions when solving simple multiplication problems. Cortex, 57, 143-155.

Brigadoi, S., Ceccherini, L., Cutini, S., Scarpa, F., Scatturin, P., Selb, J., . . Cooper, R. J. (2014). Motion artifacts in functional near-infrared spectroscopy: a comparison of motion correction techniques applied to real cognitive data. Neuroimage, 85, 181-191.

Corsi, P. M. (1973). Human memory and the medial temporal region of the brain. ProQuest Information \& Learning.

Cui, X., Bray, S., \& Reiss, A. L. (2010). Functional near infrared spectroscopy (NIRS) signal improvement based on negative correlation between oxygenated and deoxygenated hemog lobin dynamics. Neuroimage, 49(4), 3039-3046.

Davis, N., Cannistraci, C. J., Rogers, B. P., Gatenby, J. C., Fuchs, L. S., Anderson, A. W., \& Gore, J. C. (2009). Aberrant functional activation in school age children at-risk for mathematical disability: a functional imaging study of simple arithmetic skill. Neuropsychologia, 47(12), 2470-2479.

De Smedt, B., Peters, L., \& Ghesquière, P. (2019). Neurobiological origins of mathematical learning disabilities or dyscalculia: A review of brain imaging data. In A. Fritz, V. G. Haase, \& P. Räsänen (Eds.), International Handbook of Mathematical Learning Difficulties (pp. 367-384). Cham, Switzerland: Springer.

Dehaene, S., Piazza, M., Pinel, P., \& Cohen, L. (2003). Three Parietal Circuits for Number Processing. Cognitive Neuropsychology, 20(3-6), 487-506.

Dresler, T., Bugden, S., Gouet, C., Lallier, M., Oliveira, D. G., Pinheiro-Chagas, P., ... Weissheimer, J. (2018). A Translational Framework of Educational Neuroscience in Learning Disorders. Frontiers in Integrative Neuroscience, 12.

Edwards, L. A., Wagner, J. B., Simon, C. E., \& Hyde, D. C. (2016). Functional brain organization for number processing in pre-verbal infants. Developmental science, 19(5), 757-769.

Ehlert, A., Schroeders, U., \& Fritz-Stratmann, A. J. L. u. L. (2012). Criticism of the discrepancy criterion in the diagnosis of dyslexia and dyscalculia. 1(3), 169-184.

Fishburn, F. A., Ludlum, R. S., Vaidya, C. J., \& Medvedev, A. V. (2019). Temporal derivative distribution repair (TDDR): a motion correction method for fNIRS. Neuroimage, 184, 171-179. 
Gross, J., Hudson, C., \& Price, D. (2009). The long term costs of numeracy difficulties. Every Child a Chance Trust and KPMG, London.

Haeussinger, F. B., Heinzel, S., Hahn, T., Schecklmann, M., Ehlis, A.-C., \& Fallgatter, A. J. (2011). Simulation of near-infrared light absorption considering individual head and prefrontalcortex anatomy: implications for optical neuroimaging. PLoS One, 6(10), e26377.

Haeussinger, F. B., Dresler, T., Heinzel, S., Schecklmann, M., Fallgatter, A. J., \& Ehlis, A. C. (2014). Reconstructing functional near-infrared spectroscopy (fNIRS) signals impaired by extra-cranial confounds: an easy-to-use filter method. Neuroimage, 95, 69-79.

Hyde, D. C., Boas, D. A., Blair, C., \& Carey, S. (2010). Near-infrared spectroscopy shows right parietal specialization for number in pre-verbal infants. Neuroimage, 53(2), 647-652.

Iuculano, T., Rosenberg-Lee, M., Richardson, J., Tenison, C., Fuchs, L., Supekar, K., \& Menon, V. (2015). Cognitive tutoring induces widespread neuroplasticity and remediates brain function in children with mathematical learning disabilities. Nature communications, 6, 8453.

jamovi project. (2019). jamovi (Version 1). [Computer Software]. Retrieved from https://www.jamovi.org

Jolles, D., Ashkenazi, S., Kochalka, J., Evans, T., Richardson, J., Rosenberg-Lee, M., . . . Menon, V. (2016). Parietal hyper-connectivity, aberrant brain organization, and circuit-based biomarkers in children with mathematical disabilities. Developmental science, 19(4), 613-631.

Kaufmann, L., Wood, G., Rubinsten, O., \& Henik, A. (2011). Meta-analyses of developmental fMRI studies investigating typical and atypical trajectories of number processing and calculation. Developmental Neuropsychology, 36(6), 763-787.

Kaufmann, L., Mazzocco, M. M., Dowker, A., von Aster, M., Gobel, S. M., Grabner, R. H., ... Nuerk, H. C. (2013). Dyscalculia from a developmental and differential perspective. Frontiers in psychology, 4, 516.

Kesler, S. R., Sheau, K., Koovakkattu, D., \& Reiss, A. L. (2011). Changes in frontal-parietal activation and math skills performance following adaptive number sense training: preliminary results from a pilot study. Neuropsychological Rehabilitation, 21(4), 433-454.

Klein, E., Suchan, J., Moeller, K., Karnath, H. O., Knops, A., Wood, G., .. . Willmes, K. (2016). Considering structural connectivity in the triple code model of numerical cognition: differential connectivity for magnitude processing and arithmetic facts. Brain Structure and Function, 221(2), 979-995.

Kucian, K., \& von Aster, M. (2015). Developmental dyscalculia. European Journal of Pediatrics, 174(1), 113.

Kucian, K., Loenneker, T., Dietrich, T., Dosch, M., Martin, E., \& von Aster, M. (2006). Impaired neural networks for approximate calculation in dyscalculic children: a functional MRI study. Behavioral and brain functions, 2, 31.

Kucian, K., Ashkenazi, S. S., Hanggi, J., Rotzer, S., Jancke, L., Martin, E., \& von Aster, M. (2014). Developmental dyscalculia: a dysconnection syndrome? Brain Structure and Function, 219(5), 1721-1733.

Kucian, K., Grond, U., Rotzer, S., Henzi, B., Schönmann, C., Plangger, F., . . von Aster, M. (2011). Mental number line training in children with developmental dyscalculia. Neuroimage, 57(3), 782-795.

McCaskey, U., von Aster, M., Maurer, U., Martin, E., O'Gorman Tuura, R., \& Kucian, K. (2018). Longitudinal Brain Development of Numerical Skills in Typically Developing Children and Children with Developmental Dyscalculia. Frontiers in human neuroscience, 11, 629.

Michels, L., O'Gorman, R., \& Kucian, K. (2018). Functional hyperconnectivity vanishes in children with developmental dyscalculia after numerical intervention. Developmental Cognitive Neuroscience, 30, 291-303. 
Molko, N., Cachia, A., Rivière, D., Mangin, J. F., Bruandet, M., LeBihan, D., ... Dehaene, S. (2003). Functional and structural alterations of the intraparietal sulcus in a developmental dyscalculia of genetic origin. Neuron, 40(4), 847-858.

Molko, N., Cachia, A., Riviere, D., Mangin, J. F., Bruandet, M., LeBihan, D., ... Dehaene, S. (2004). Brain Anatomy in Turner Syndrome: Evidence for Impaired Social and Spatial-Numerical Networks. Cerebral cortex, 14(8), 840-850.

Moser Opitz, E., Ramseier, E., Reusser, L., Haselhorn, M., Heinzel, A., Schneider, W., \& Trautwein, U. (2010). Basisdiagnostik Mathematik für die Klassen 4-8 (BASIS-Math 4-8). Jahrbuch der pädagogisch-psychologischen Diagnostik. Tests und Trends. Neue Folge(11), 271-286.

Petermann, F., Petermann, U., \& Wechsler, D. (2007). Hamburg-Wechsler-Intelligenztest für Kinder-IV: HAWIK-IV: Huber.

Peters, L., \& De Smedt, B. (2018). Arithmetic in the developing brain: A review of brain imaging studies. Developmental Cognitive Neuroscience, 30, 265-279.

Peters, L., Bulthe, J., Daniels, N., Op de Beeck, H., \& De Smedt, B. (2018). Dyscalculia and dyslexia: Different behavioral, yet similar brain activity profiles during arithmetic. Neurolmage: Clinical, 18, 663-674.

Polspoel, B., Peters, L., Vandermosten, M., \& De Smedt, B. (2017). Strategy over operation: neural activation in subtraction and multiplication during fact retrieval and procedural strategy use in children. Human brain mapping, 38(9), 4657-4670.

R Core Team. (2018). R: A Language and Environment for Statistical Computing. Vienna, Austria. Retrieved from https://www.R-project.org/

Ranpura, A., Isaacs, E., Edmonds, C., Rogers, M., Lanigan, J., Singhal, A., . . Butterworth, B. (2013). Developmental trajectories of grey and white matter in dyscalculia. Trends in Neuroscience and Education, 2(2), 56-64.

Rorden, C., \& Brett, M. (2000). Stereotaxic display of brain lesions. Behavioural neurology, 12(4), 191200.

Rosenberg-Lee, M., Ashkenazi, S., Chen, T., Young, C. B., Geary, D. C., \& Menon, V. (2015). Brain hyperconnectivity and operation-specific deficits during arithmetic problem solving in children with developmental dyscalculia. Developmental science, 18(3), 351-372.

Rotzer, S., Kucian, K., Martin, E., von Aster, M., Klaver, P., \& Loenneker, T. (2008). Optimized voxel-based morphometry in children with developmental dyscalculia. Neuroimage, 39(1), 417-422.

Rykhlevskaia, E., Uddin, L. Q., Kondos, L., \& Menon, V. (2009). Neuroanatomical correlates of developmental dyscalculia: combined evidence from morphometry and tractography. Frontiers in human neuroscience, 3, 51.

Sasai, S., Homae, F., Watanabe, H., \& Taga, G. (2011). Frequency-specific functional connectivity in the brain during resting state revealed by NIRS. Neuroimage, 56(1), 252-257.

Scholkmann, F., Kleiser, S., Metz, A. J., Zimmermann, R., Pavia, J. M., Wolf, U., \& Wolf, M. (2014). A review on continuous wave functional near-infrared spectroscopy and imaging instrumentation and methodology. Neuroimage, 85, 6-27.

Schroeter, M. L., Kupka, T., Mildner, T., Uludağ, K., \& von Cramon, D. Y. (2006). Investigating the poststimulus undershoot of the BOLD signal-a simultaneous $\mathrm{fMRI}$ and fNIRS study. Neuroimage, 30(2), 349-358.

Schwartz, F., Epinat-Duclos, J., Leone, J., Poisson, A., \& Prado, J. (2018). Impaired neural processing of transitive relations in children with math learning difficulty. Neurolmage: Clinical, 20, 12551265.

Simos, P. G., Kanatsouli, K., Fletcher, J. M., Sarkari, S., Juranek, J., Cirino, P., . . Papanicola ou, A. C. (2008). Aberrant spatiotemporal activation profiles associated with math difficulties in children: a magnetic source imaging study. Neuropsychology, 22(5), 571-584. 
Singh, A. K., Okamoto, M., Dan, H., Jurcak, V., \& Dan, I. (2005). Spatial registration of multichannel multisubject fNIRS data to MNI space without MRI. Neuroimage, 27(4), 842-851.

Soltanlou, M., Sitnikova, M. A., Nuerk, H.-C., \& Dresler, T. (2018). Applications of functional near-Infrared spectroscopy (fNIRS) in studying cognitive development: The case of mathematics and language. Frontiers in psychology, 9, 277.

Soltanlou, M., Artemenko, C., Dresler, T., Haeussinger, F. B., Fallgatter, A. J., Ehlis, A. C., \& Nuerk, H. C. (2017). Increased arithmetic complexity is associated with domain-general but not domainspecific magnitude processing in children: A simultaneous fNIRS-EEG study. Cognitive, Affective, \& Behavioral Neuroscience, 17(4), 724-736.

Soltanlou, M., Artemenko, C., Ehlis, A. -C., Huber, S., Fallgatter, A. J., Dresler, T., \& Nuerk, H. -C. (2018). Reduction but no shift in brain activation after arithmetic learning in children: A simultaneous fNIRS-EEG study. Scientific reports, 8(1), 1707.

Soltanlou, M., Jung, S., Roesch, S., Ninaus, M., Brandelik, K., Heller, J., . . Moeller, K. (2017). Behavioral and neurocognitive evaluation of a web-platform for game-based learning of orthography and numeracy Informational Environments (pp. 149-176): Springer.

Tong, Y., \& Frederick, B. D. (2010). Time lag dependent multimodal processing of concurrent fMRI and near-infrared spectroscopy (NIRS) data suggests a global circulatory origin for low-frequency oscillation signals in human brain. Neuroimage, 53(2), 553-564.

Tsuzuki, D., Jurcak, V., Singh, A. K., Okamoto, M., Watanabe, E., \& Dan, I. (2007). Virtual spatial registration of stand-alone fNIRS data to MNI space. Neuroimage, 34(4), 1506-1518.

Tzourio-Mazoyer, N., Landeau, B., Papathanassiou, D., Crivello, F., Etard, O., Delcroix, N., ... Joliot, M. (2002). Automated anatomical labeling of activations in SPM using a macroscopic anatomical parcellation of the MNI MRI single-subject brain. Neuroimage, 15(1), 273-289.

Vogel, S. E., Goffin, C., \& Ansari, D. (2015). Developmental specialization of the left parietal cortex for the semantic representation of Arabic numerals: an fMR-adaptation study. Developmental Cognitive Neuroscience, 12, 61-73.

Wimmer, H., \& Mayringer, H. (2014). SLS 2-9: Salzburger Lese-Screening für die Schulstufen 2-9. Göttingen: Huber, Hogrefe. 


\section{Supplementary Materials for}

\section{Training causes activation increase in parietal and temporo-parietal regions in children with developmental dyscalculia}

Mojtaba Soltanlou, Thomas Dresler, Christina Artemenko, David Rosenbaum, Ann-Christine Ehlis, Hans-Christoph Nuerk

Correlation between demographic information and neuropsychological tests

Pearson correlation was conducted between demographic information and neuropsychological data (Table S1). The correlation analysis revealed that children in higher grades, who are older, had better non-verbal IQ and mathematics knowledge. Children who had better verbal IQ, had better verbal working memory as well. Children who had better non-verbal IQ, had better visuospatial short-term memory and mathematics knowledge.

Table S1: Pearson correlations between demographic information and neuropsychological data.

\begin{tabular}{lrrrrrrrrr}
\hline & $\mathbf{1}$ & $\mathbf{2}$ & $\mathbf{3}$ & $\mathbf{4}$ & $\mathbf{5}$ & $\mathbf{6}$ & $\mathbf{7}$ & $\mathbf{8}$ & $\mathbf{9}$ \\
\hline 1. Age & - & & & & & & & & \\
2. Grade & $\mathbf{0 . 8 3}$ & - & & & & & & & \\
3. Verbal IQ & -0.07 & 0.06 & - & & & & & & \\
4. Non-verbal IQ & 0.23 & $\mathbf{0 . 4 8}$ & 0.44 & - & & & & & \\
5. Verbal short-termmemory & 0.17 & 0.09 & -0.14 & -0.17 & - & & & \\
6. Verbal working memory & 0.01 & 0.15 & $\mathbf{0 . 4 5}$ & 0.44 & -0.40 & - & & \\
7. Visuospatial short-termmemory & 0.20 & 0.18 & -0.08 & $\mathbf{0 . 5 4}$ & -0.31 & 0.16 & - & & \\
8. Visuospatial working memory & 0.03 & 0.24 & -0.36 & 0.07 & -0.04 & 0.13 & 0.16 & - & \\
9. Reading & -0.22 & 0.03 & 0.14 & 0.21 & 0.01 & 0.11 & -0.05 & -0.17 & - \\
10. Mathematics knowledge & 0.39 & $\mathbf{0 . 5 4}$ & 0.41 & $\mathbf{0 . 5 5}$ & 0.00 & 0.44 & 0.16 & 0.19 & 0.22 \\
\hline
\end{tabular}

Note: $N=20$ (but 19 for the letter span - backward, and 18 for the reading test); uncorrected $p<$ .05 , two-tailed; significant results are shown in bold. 


\section{Report of rmANCOVAs on behavioral and fNIRS data}

Table S2: Behavioral results of rmANCOVAs of median RT and error rate for simple and complex multiplication.

\begin{tabular}{|c|c|c|c|c|c|c|}
\hline & \multicolumn{3}{|c|}{ Simple } & \multicolumn{3}{|c|}{ Complex } \\
\hline & $F$ & $p$ & $\overline{\eta_{p}{ }^{2}}$ & $F$ & $\bar{p}$ & $\overline{\eta_{p}{ }^{2}}$ \\
\hline \multicolumn{7}{|l|}{ Median RT } \\
\hline Time & 0.01 & .995 & .001 & 5.39 & .032 & .230 \\
\hline Time $\times$ Grade & 0.14 & .712 & .008 & 3.47 & .079 & .162 \\
\hline Training & 2.63 & .122 & .128 & 4.59 & .046 & .203 \\
\hline Training $\times$ Grade & 1.64 & .216 & .084 & 2.65 & .121 & .128 \\
\hline Time $\times$ Training & 0.36 & .559 & .019 & 0.91 & .352 & .048 \\
\hline Time $\times$ Training $\times$ Grade & 1.02 & .326 & .054 & 1.00 & .331 & .052 \\
\hline Grade & 3.29 & .086 & .155 & 14.70 & .001 & .450 \\
\hline \multicolumn{7}{|l|}{ Error rate } \\
\hline Time & 1.02 & .327 & .053 & 4.39 & .050 & .196 \\
\hline Time $\times$ Grade & 1.64 & .217 & .084 & 3.56 & .075 & .165 \\
\hline Training & 6.52 & .020 & .266 & 0.19 & .666 & .011 \\
\hline Training $\times$ Grade & 4.90 & .040 & .214 & 0.07 & .800 & .004 \\
\hline Time $\times$ Training & 2.50 & .132 & .122 & 0.21 & .650 & .012 \\
\hline Time $\times$ Training $\times$ Grade & 2.20 & .155 & .109 & 0.07 & .795 & .004 \\
\hline Grade & 2.35 & .142 & .116 & 11.60 & .003 & .391 \\
\hline
\end{tabular}

Note: $N=20 ; d f=(1,18)$; significant results are shown in bold.

Table S3: fNIRS results of rmANCOVAs for simple multiplication.

\begin{tabular}{l|ccc|ccc|ccc}
\hline & \multicolumn{3}{|c|}{ Frontal } & \multicolumn{3}{c|}{ Parietal } & \multicolumn{3}{c}{ Temporo-parietal } \\
\hline Time & $F$ & $p$ & $\eta_{p}{ }^{2}$ & $F$ & $p$ & $\eta_{p}{ }^{2}$ & $F$ & $p$ & $\eta_{p}{ }^{2}$ \\
\hline Time $\times$ Grade & 1.27 & .275 & .066 & 1.88 & .187 & .095 & 1.90 & .185 & .095 \\
\hline Training & 1.27 & .275 & .066 & 2.02 & .172 & .101 & 1.76 & .201 & .089 \\
\hline Training $\times$ Grade & 2.04 & .170 & .102 & 1.69 & .210 & .086 & 0.73 & .404 & .039 \\
\hline Hemis phere & 2.21 & .155 & .109 & 1.96 & .178 & .098 & 0.71 & .411 & .038 \\
\hline Hemisphere $\times$ Grade & 0.28 & .602 & .015 & 0.07 & .789 & .004 & 2.81 & .111 & .135 \\
\hline Time $\times$ Training & 0.49 & .495 & .026 & 0.22 & .648 & .012 & 2.40 & .139 & .118 \\
\hline Time $\times$ Training $\times$ Grade & 0.14 & .715 & .008 & 1.79 & .197 & .091 & 4.72 & .043 & .208 \\
\hline Time $\times$ Hemis phere & 0.09 & .770 & .005 & 1.62 & .220 & .082 & 3.95 & .062 & .180 \\
\hline Time $\times$ Hemisphere $\times$ Grade & 0.97 & .338 & .051 & 0.13 & .720 & .007 & 1.64 & .216 & .084 \\
\hline Training $\times$ Hemisphere & 0.42 & .527 & .023 & 0.08 & .778 & .005 & 1.72 & .206 & .087 \\
\hline Training $\times$ Hemisphere $\times$ Grade & 0.21 & .654 & .011 & 0.07 & .794 & .004 & 1.51 & .235 & .077 \\
\hline
\end{tabular}




\begin{tabular}{l|ccc|ccc|ccc}
\hline Time $\times$ Training $\times$ Hemisphere & 0.04 & .850 & .002 & 0.21 & .649 & .012 & 0.95 & .342 & .050 \\
\hline $\begin{array}{l}\text { Time } \times \text { Training } \times \text { Hemisphere } \times \\
\text { Grade }\end{array}$ & 0.15 & .704 & .008 & 0.48 & .497 & .026 & 0.69 & .416 & .037 \\
\hline Grade & 0.36 & .557 & .020 & 1.41 & .250 & .073 & 1.54 & .231 & .079 \\
\hline
\end{tabular}

Note: $N=20 ; d f=(1,18)$; significant results are shown in bold.

Table S4: fNIRS results of rmANCOVAs for complex multiplication.

\begin{tabular}{|c|c|c|c|c|c|c|c|c|c|}
\hline & \multicolumn{3}{|c|}{ Frontal } & \multicolumn{3}{|c|}{ Parietal } & \multicolumn{3}{|c|}{ Temporo-parietal } \\
\hline & $F$ & $p$ & $\overline{\eta_{p}{ }^{2}}$ & $F$ & $p$ & $\overline{\eta_{p}{ }^{2}}$ & $F$ & $\bar{p}$ & $\eta_{p}{ }^{2}$ \\
\hline Time & 6.46 & .020 & .264 & 1.31 & .268 & .068 & 6.21 & .023 & .256 \\
\hline Time $\times$ Grade & 5.42 & .032 & .231 & 0.92 & .350 & .049 & 4.35 & .052 & .194 \\
\hline Training & 0.32 & .577 & .018 & 0.06 & .814 & .003 & 0.48 & .499 & .026 \\
\hline Training $\times$ Grade & 0.29 & .596 & .016 & 0.12 & .731 & .007 & 0.83 & .374 & .044 \\
\hline Hemisphere & 1.79 & .197 & .091 & 5.14 & .036 & .222 & 1.17 & .293 & .061 \\
\hline Hemis phere $\times$ Grade & 1.73 & .205 & .088 & 5.20 & .035 & .224 & 0.72 & .406 & .039 \\
\hline Time $\times$ Training & 1.48 & .239 & .076 & 5.63 & .029 & .238 & 0.87 & .363 & .046 \\
\hline Time $\times$ Training $\times$ Grade & 1.75 & .202 & .089 & 5.12 & .036 & .221 & 0.72 & .406 & .039 \\
\hline Time $\times$ Hemis phere & 6.37 & .021 & .261 & 0.63 & .439 & .034 & 0.01 & .928 & .000 \\
\hline Time $\times$ Hemisphere $\times$ Grade & 5.77 & .027 & .243 & 0.70 & .424 & .036 & 0.04 & .843 & .002 \\
\hline Training $\times$ Hemis phere & 0.06 & .815 & .003 & 0.01 & .978 & .001 & 0.56 & .464 & .030 \\
\hline Training $\times$ Hemis phere $\times$ Grade & 0.05 & .822 & .003 & 0.07 & .791 & .004 & 0.79 & .385 & .042 \\
\hline Time $\times$ Training $\times$ Hemis phere & 0.84 & .371 & .045 & 3.38 & .082 & .158 & 1.51 & .235 & .078 \\
\hline $\begin{array}{l}\text { Time } \times \text { Training } \times \text { Hemis phere } \times \\
\text { Grade }\end{array}$ & 0.81 & .380 & .043 & 3.31 & .085 & .155 & 2.16 & .159 & .107 \\
\hline Grade & 0.70 & .415 & .037 & 0.23 & .641 & .012 & 0.98 & .336 & .051 \\
\hline
\end{tabular}

Note: $N=20 ; d f=(1,18)$; significant results are shown in bold.

Table S5: fNIRS results of the rmANCOVA for global analysis.

\begin{tabular}{l|ccc}
\hline & $\boldsymbol{F}$ & $\boldsymbol{p}$ & $\boldsymbol{\eta}^{2}$ \\
\hline Time & 0.52 & .479 & .028 \\
\hline Time $\times$ Grade & 0.29 & .599 & .016 \\
\hline Complexity & 2.21 & .155 & .109 \\
\hline Complexity $\times$ Grade & 2.53 & .129 & .123 \\
\hline Time $\times$ Complexity & 4.84 &. $\mathbf{0 4 1}$ & .212 \\
\hline Time $\times$ Complexity $\times$ Grade & 3.78 & .068 & .173 \\
\hline Grade & 0.01 & .930 & .001 \\
\hline
\end{tabular}

Note: $N=20 ; d f=(1,18)$; significant results are shown in bold. 


\section{Behavioral training effects}

Similar to Kucian et al. (2011), some additional tests were administered before and after training. The tests included a general arithmetic ability test, a strategy questionnaire (Soltanlou et al., 2018), and information about self-concept, attitude towards math, and concerns about math (Soltanlou et al., 2019) (Table S6). To uncover the generalization and transfer effect of the twoweek multiplication training in the above-mentioned tasks, paired $t$-tests (post-test versus pretest) were conducted (Table S6).

The arithmetic ability test contained four basic operations (addition, subtraction, multiplication, and division) with two difficulty levels (simple and complex). Children were asked to solve as many problems as they could in a given time. They had $45 \mathrm{~s}$ for each list of simple problems and $60 \mathrm{~s}$ for each list of complex problems. Children showed better arithmetic performance (i.e., more correctly solved problems) after two weeks of training. This improved performance was significant for simple multiplication (near transfer), and complex subtraction and division (far transfer; Table S6).

The strategy questionnaire consisted of two multiplication problems from each of the four experimental conditions (simple/complex and trained/untrained) and was provided in four matched versions without a time limit. The children reported how they came to the solution after each problem. According to the child's report, experimenters categorized each strategy as retrieval, procedural, or other (Soltanlou et al., 2018). Interestingly, children with DD reported higher utilization of the retrieval strategy after training (Table S6).

As it has been explained by Soltanlou et al. (2019), self-concept, attitude towards math, and concerns about math are part of the German translation of the math anxiety questionnaire (MAQ, Krinzinger et al., 2007; the original English version by Thomas \& Dowker, 2000), which 
has an internal consistency (Cronbach's alpha) of $0.83-0.91$ for the whole questionnaire for different age groups. Each subscale contains five items describing different math-related topics. Each item needs to be rated on a five-point Likert scale ( 0 to 4$)$ with a maximum score of 20 on each subscale. Thereby, higher values indicate a higher self-concept, a more positive attitude towards math, and less concern about math, respectively. While the self-concept in math and the attitude towards math subscales demonstrate general math-related attitudes, the concerns subscale about math indicates math anxiety (Krinzinger, Kaufmann, \& Willmes, 2009). Children with DD reported a higher self-concept in math and lower math anxiety after two weeks of training (Table S6).

Table S6: Pre- and post-test comparisons for arithmetic ability (addition, subtraction, multiplication, division), strategy use, and math anxiety.

\begin{tabular}{llrrrrrrrr}
\hline & \multicolumn{2}{c}{ Pre-test } & \multicolumn{2}{c}{ Post-test } & Range & $\boldsymbol{t}(\mathbf{1 9})$ & $\boldsymbol{p}$ & $\boldsymbol{d}$ \\
& & Mean & SD & Mean & SD & & & & \\
\hline Addition & Simple & 10.40 & 2.41 & 10.20 & 2.14 & $0-18$ & -0.48 & .680 & -0.11 \\
& Complex & 8.20 & 2.31 & 7.95 & 2.04 & $0-18$ & -0.68 & .747 & -0.15 \\
Subtraction & Simple & 8.30 & 2.23 & 8.45 & 2.19 & $0-15$ & 0.30 & .383 & 0.07 \\
& Complex & 4.60 & 2.14 & 5.50 & 2.06 & $0-15$ & 2.10 & $\mathbf{. 0 2 5}$ & 0.47 \\
Multiplication & Simple & & & & & & & & \\
& Complex & 2.65 & 4.04 & 8.90 & 3.91 & $0-20$ & 2.16 & $\mathbf{. 0 2 2}$ & 0.48 \\
& & 1.73 & 2.80 & 1.85 & $0-13$ & 1.05 & .154 & 0.23 \\
Division & Simple & 8.65 & 5.15 & 9.15 & 4.68 & $0-28$ & 0.64 & .266 & 0.14 \\
& Complex & 2.00 & 1.65 & 2.75 & 1.83 & $0-14$ & 2.68 & $\mathbf{. 0 0 7}$ & 0.60 \\
Strategy & & & & & & & & & \\
& Retrieval & 2.40 & 1.27 & 3.35 & 1.35 & $0-8$ & 2.76 & $\mathbf{. 0 0 6}$ & 0.62 \\
Math anxiety & Procedural & 4.45 & 1.90 & 4.10 & 1.33 & $0-8$ & -0.73 & .239 & -0.16 \\
& Self-concept & 11.45 & 3.52 & 12.30 & 3.48 & $0-20$ & 2.74 & $\mathbf{. 0 0 6}$ & 0.61 \\
& Attitude & 11.15 & 3.17 & 11.75 & 3.09 & $0-20$ & 1.50 & .075 & 0.34 \\
& Anxiety & 6.10 & 3.06 & 7.00 & 2.88 & $0-20$ & 1.81 & $\mathbf{. 0 4 3}$ & 0.41 \\
\hline
\end{tabular}

Note: $N=20$; uncorrected $p<.05$, one-tailed; significant results are shown in bold. 


\section{Experimental trials}

The stimulus set consisted of 32 multiplication problems with 8 problems in each of the 4 condition (Soltanlou et al., 2018).

Table S7: Multiplication stimulus sets per condition.

\begin{tabular}{cccc}
\hline Trained simple & Trained complex & Untrained simple & Untrained complex \\
\hline $3 \times 4$ & $13 \times 4$ & $6 \times 2$ & $18 \times 3$ \\
$5 \times 3$ & $3 \times 19$ & $7 \times 2$ & $6 \times 12$ \\
$2 \times 8$ & $5 \times 13$ & $3 \times 7$ & $4 \times 19$ \\
$6 \times 3$ & $18 \times 4$ & $4 \times 6$ & $7 \times 12$ \\
$3 \times 9$ & $6 \times 13$ & $8 \times 3$ & $14 \times 6$ \\
$7 \times 4$ & $15 \times 6$ & $7 \times 5$ & $17 \times 5$ \\
$5 \times 6$ & $12 \times 8$ & $4 \times 9$ & $5 \times 18$ \\
$8 \times 4$ & $7 \times 14$ & $5 \times 8$ & $13 \times 7$ \\
\hline
\end{tabular}

\section{References}

Krinzinger, H., Kaufmann, L., \& Willmes, K. (2009). Math anxiety and math ability in early primary school years. Journal of psychoeducational assessment, 27(3), 206-225.

Krinzinger, H., Kaufmann, L., Dowker, A., Thomas, G., Graf, M., Nuerk, H. -C., \& Willmes, K. (2007). Deutschsprachige Version des Fragebogens für Rechenangst (FRA) für 6-bis 9-jährige Kinder. Zeitschrift für Kinder-und Jugendpsychiatrie und Psychotherapie, 35(5), 341-351.

Kucian, K., Grond, U., Rotzer, S., Henzi, B., Schönmann, C., Plangger, F., . . von Aster, M. (2011). Mental number line training in children with developmental dyscalculia. Neuroimage, 57(3), 782-795.

Soltanlou, M., Artemenko, C., Dresler, T., Fallgatter, A. J., Ehlis, A. C., \& Nuerk, H. C. (2019). Math Anxiety in Combination With Low Visuospatial Memory Impairs Math Learning in Children. Frontiers in psychology, 10, 89.

Soltanlou, M., Artemenko, C., Ehlis, A.-C., Huber, S., Fallgatter, A. J., Dresler, T., \& Nuerk, H. -C. (2018). Reduction but no shift in brain activation after arithmetic learning in children: $A$ simultaneous fNIRS-EEG study. Scientific reports, 8(1), 1707.

Thomas, G., \& Dowker, A. (2000). Mathematics anxiety and related factors in young children. Paper presented at the British Psychological Society Developmental Section Conference, Bristol, UK. 